\title{
Unusual Temperature Dependence of Enantio-selectivity in Asymmetric Reductions by Chiral NADH Models
}

\author{
Ryota Saito, ${ }^{*},{ }^{\dagger}$ Shoichiro Naruse ${ }^{\dagger}$ Koji Takano, ${ }^{\dagger}$ Keiko \\ Fukuda, ${ }^{\dagger}$ Akira Katoh, ${ }^{\dagger}$ and Yoshihisa Inoue $*, \$$
}

\begin{abstract}
$\uparrow$ Department of Materials and Life Science, Seikei University, 3-3-1 Kichijoji-kitamachi, Musashino 180-8633, Japan. $¥$ Entropy Control Project (ICORP), JST, Kamishinden, Toyonaka 560-0085, Japan. §Department of Applied Chemistry, Osaka University, 2-1 Yamada-oka, Suita 565-0871, Japan.
\end{abstract}

\section{Experimental}

General. All melting points were measured on a Yamato MP-21 or a Laboratory Devices Mel-Temp II apparatuses in open capillaries and are uncorrected. ${ }^{1} \mathrm{H}-\mathrm{NMR}$ spectra were recorded on JEOL JNM-LA400 (400 MHz) spectrometers. Chemical shifts $(\delta)$ are reported in ppm using tetramethylsilane or an undeuteriated solvent as internal standards in a deuteriated solvent used. Coupling constants $(J)$ are given in Hz. Infrared spectra were obtained using JASCO FT/IR-230 or FT/IR-470plus spectrophotometers. Absorption spectra were measured on a Jasco V-550 spectrophotometer. Combustion analyses were performed on a Yanaco MT-3 CHN Corder or a Perkin Elmer Series II CHNS/O Analyzer 2400. Column chromatography was carried out on silica gel $(63-210 \mathrm{~mm}$ particle size, Kanto Chemical Co.). Gas chromatographic (GC) analyses were performed on a J\&W Scientific DB-5MS capillary column $(0.32 \mathrm{~mm}$ I.D. $x 30 \mathrm{~m})$ at $110{ }^{\circ} \mathrm{C}$, using a Shimadzu GC-8A or GC-14B instrument equipped with a C-R6A integrator. High performance liquid chromatography (HPLC) analyses were performed on a Daicel Chiralcel OJ column (4.6 mm I.D. x $250 \mathrm{~nm}$ ), using a JASCO PU-980 HPLC pump and UV-970 UV/VIS detector controlled by a JASCO LCSS-905 System Station. Optical rotations were recorded on a Jasco DIP-1000 Polarimeter.

All conventional chemicals used in the present study were commercially available. Methyl bonzoylformate was purified by distillation prior to use. Other chemicals were used as received. 
Synthesis. 1,4-Dihydronicotinamides (1) bearing amino acid methyl esters, L-alanine (Ala-OMe), L-valine (Val-OMe), L-phenylalanine (Phe-OMe), L-proline (Pro-OMe) and (S)-phenylglycine (PhG-OMe) methyl esters, were synthesized by reduction of the corresponding pyridinium salts, which were synthesized by reacting nicotinyl chloride hydrochloride with methyl esters of the corresponding amino acids in the presence of triethyl amine followed by $N$-methylation with iodomethane (Scheme 1). ${ }^{1}$

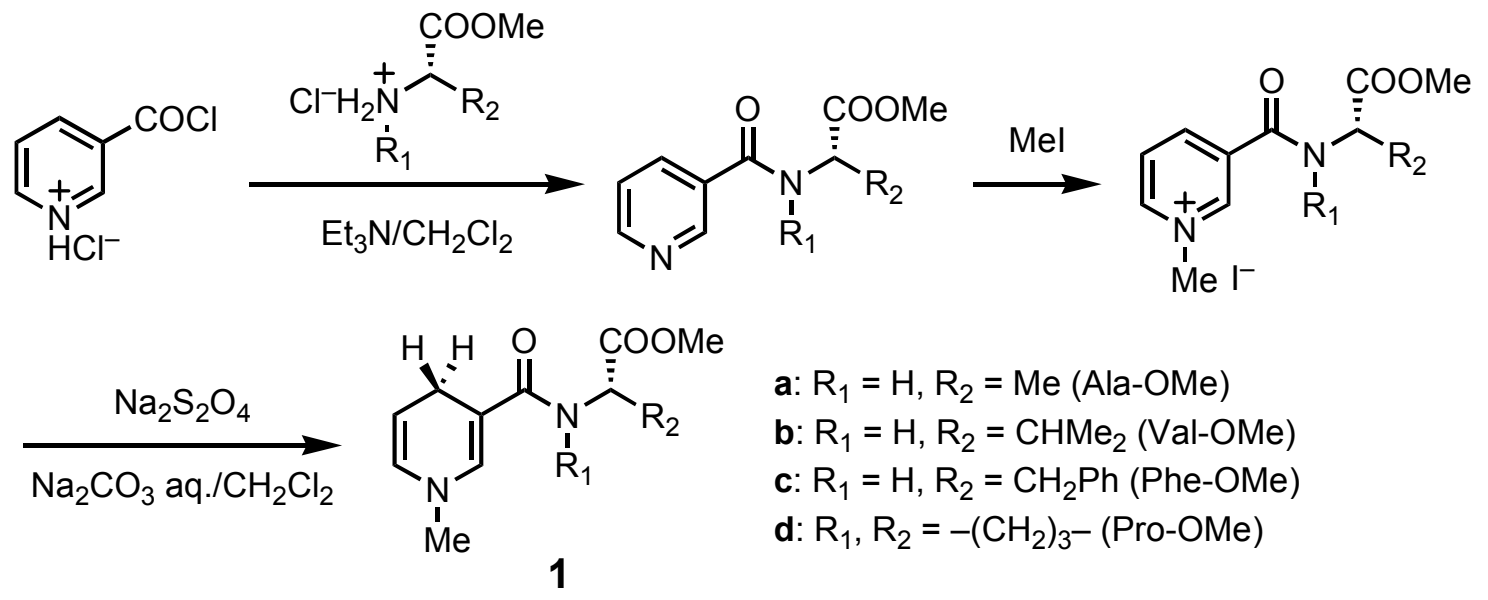

Scheme S1. Preparation of 1,4-dihydropyridines possessing amino acid esters as chiral auxiliaries.

Representative procedures for the synthesis of $N$-nicotonyl amino acid methyl esters: $\mathrm{N}$-Nicotinylalanine methyl ester (Nic-Ala-OMe). To a solution of alanine methyl ester hydrochloride (698 mg, $5.00 \mathrm{mmol}$ ) and triethylamine (1.52 g, $15.0 \mathrm{mmol})$ in dichloromethane $(100 \mathrm{~mL})$ was added slowly nicotinyl chloride hydrochloride $(890 \mathrm{mg}, 5.00 \mathrm{mmol})$ at $0{ }^{\circ} \mathrm{C}$. The mixture was then stirred at $0{ }^{\circ} \mathrm{C}$ for $2 \mathrm{~h}$. The reaction mixture was successively washed with $5 \%$ aqueous sodium bicarbonate $(100 \mathrm{~mL} \times 2)$, and with $5 \%$ aqueous citric acid $(100 \mathrm{~mL} \times$ 2 ). The organic layer was washed with brine and then dried over sodium sulfate. After removal of the solvent, the crude product was purified by column chromatography on silica gel with ethyl acetate as an eluent to afford pure product as pale yellow needles (789 $\mathrm{mg}, 76 \%$ ), mp 73-74 ${ }^{\circ} \mathrm{C}:[\alpha]^{24}+42.6^{\circ}\left(c 0.59, \mathrm{CHCl}_{3}\right)$; IR $(\mathrm{KBr}) v_{\max } / \mathrm{cm}^{-1} 3326(v \mathrm{NH}), 1744,1639(v \mathrm{C}=\mathrm{O})$, 1215, 1172 (vC-O); ${ }^{1} \mathrm{H}$ NMR $\left(\mathrm{CDCl}_{3}, 400 \mathrm{MHz}\right) \delta 9.03(1 \mathrm{H}, \mathrm{d}, J=2.0, \mathrm{py}-2-H), 8.75(1 \mathrm{H}, \mathrm{dd}$, $J=2.0$ and 4.9, py-6- $H), 8.13(1 \mathrm{H}, \mathrm{td}, J=2.0$ and 8.0, py-4- $H), 7.41(1 \mathrm{H}, \mathrm{dd}, J=4.9$ and 8.0, py-5- $H), 6.83(1 \mathrm{H}, \mathrm{d}, J=5.8, \mathrm{~N} H), 4.82\left(1 \mathrm{H}\right.$, quintet, $\left.J=7.1, \mathrm{CHCH}_{3}\right), 3.81\left(3 \mathrm{H}, \mathrm{s}, \mathrm{OCH}_{3}\right)$, $1.55\left(3 \mathrm{H}, \mathrm{d}, J=7.1, \mathrm{CHCH}_{3}\right)$. Anal. Calcd for $\mathrm{C}_{10} \mathrm{H}_{12} \mathrm{~N}_{2} \mathrm{O}_{3}: \mathrm{C}: 57.69 ; \mathrm{H}: 5.81 ; \mathrm{N}: 13.45$. Found: C: 57.46; H: 5.88; N: 13.32 .<smiles>CC(=O)[C@H](C)NC(=O)c1cccnc1</smiles>

Nic-Ala-OMe 
$\mathrm{N}$-Nicotinylvaline methyl ester (Nic-Val-OMe). Yellow solid (1.52 g, 92\%), mp 99-102 ${ }^{\circ} \mathrm{C}$ : $[\alpha]^{24}+48.3^{\circ}\left(c\right.$ 0.50, $\left.\mathrm{CHCl}_{3}\right)$; IR (KBr) $v_{\max } / \mathrm{cm}^{-1} 3333(v \mathrm{NH}), 1737,1641(\mathrm{vC}=\mathrm{O}), 1204$, 1155 (vC-O); ${ }^{1} \mathrm{H}$ NMR $\left(\mathrm{CDCl}_{3}, 400 \mathrm{MHz}\right) \delta 9.04(1 \mathrm{H}, \mathrm{d}, J=1.7 \mathrm{~Hz}, \mathrm{py}-2-H), 8.76(1 \mathrm{H}, \mathrm{dt}, J$ $=4.9$ and 1.7, py-6- $H), 8.13(1 \mathrm{H}, \mathrm{dt}, J=8.1$ and 1.7, py-4- $H), 7.41(1 \mathrm{H}, \mathrm{dd}, J=8.1$ and 4.9, py-5- $H), 6.73(1 \mathrm{H}, \mathrm{d}, J=8.5, \mathrm{~N} H), 4.80(1 \mathrm{H}, \mathrm{dd}, J=8.5$ and $4.9, \mathrm{CHCO}), 3.80\left(3 \mathrm{H}, \mathrm{s}, \mathrm{OCH}_{3}\right)$, 2.25-2.35 (1 H, m, CHMe $), 1.03\left(3 \mathrm{H}, \mathrm{d}, J=6.8, \mathrm{CH}_{3}\right), 1.00\left(3 \mathrm{H}, \mathrm{d}, J=6.8, \mathrm{CH}_{3}\right)$. Anal. Calcd for $\mathrm{C}_{12} \mathrm{H}_{16} \mathrm{~N}_{2} \mathrm{O}_{3}$ : C, 61.00; H, 6.83; N, 11.86. Found: C, 60.82; H, 6.86; N, 11.86.<smiles>CC(=O)[C@H](NC(=O)c1cccnc1)C(C)C</smiles>

Nic-Val-OMe

$\mathrm{N}$-Nicotinylphenylalanine methyl ester (Nic-Phe-OMe). Yellow solid (1.34 g, 96\%), mp $70-72{ }^{\circ} \mathrm{C}:[\alpha]^{25}+93.0\left(c 0.62, \mathrm{CHCl}_{3}\right)$; IR $(\mathrm{KBr}) v_{\max } / \mathrm{cm}^{-1} 3329(\mathrm{vNH}), 1737,1640(\mathrm{vC}=\mathrm{O})$, 1219, 1162 (vC-O); ${ }^{1} \mathrm{H}$ NMR $\left(\mathrm{CDCl}_{3}, 400 \mathrm{MHz}\right) \delta 8.91(1 \mathrm{H}, \mathrm{d}, J=1.7, \mathrm{py}-2-H), 8.72(1 \mathrm{H}$, $\mathrm{dd}, J=4.9$ and 1.7, py-6- $H), 8.05(1 \mathrm{H}, \mathrm{dd}, J=8.0$ and 1.7 , py-4- $H), 7.38(1 \mathrm{H}, \mathrm{dd}, J=8.0$ and 4.9, py-5-H), 7.33-7.24 (3 H, m, Ph-3',4',5'-H), 7.13 (2 H, d, J=7.3, Ph-2',6'-H), 6.72 (1 H, d, $J=7.6, \mathrm{NH}) 5.10(1 \mathrm{H}, \mathrm{dt}, J=7.6$ and 5.6, $\mathrm{CH}), 3.79\left(3 \mathrm{H}, \mathrm{s}, \mathrm{CH}_{3}\right), 3.31$ and $3.23(2 \mathrm{H}, \mathrm{ABq}, J$ $=13.9$ and 5.6, $\mathrm{CH}_{2} \mathrm{Ph}$ ). Anal. Calcd for $\mathrm{C}_{16} \mathrm{H}_{16} \mathrm{~N}_{2} \mathrm{O}_{3}: \mathrm{C}, 67.59 ; \mathrm{H}, 5.67 ; \mathrm{N}, 9.85$. Found: $\mathrm{C}$, 67.52; H, 5.75; N, 9.72 .<smiles>CC(=O)[C@H](Cc1ccccc1)NC(=O)c1cccnc1</smiles>

Nic-Phe-OMe

$\boldsymbol{N}$-Nicotinylproline methyl ester (Nic-Pro-OMe). Yellow solid (827 $\mathrm{mg}, 71 \%$ ), mp 58-60 ${ }^{\circ} \mathrm{C}:[\alpha]^{24}{ }_{\mathrm{D}}-42.6\left(c 0.59, \mathrm{CHCl}_{3}\right)$; IR $(\mathrm{KBr}) v_{\max } / \mathrm{cm}^{-1} 1742,1628(\mathrm{vC}=\mathrm{O}), 1204,1174$ (vC-O); ${ }^{1} \mathrm{H}$ NMR $\left(\mathrm{CDCl}_{3}, 400 \mathrm{MHz}\right) \delta 8.84(1 \mathrm{H}, \mathrm{d}, J=1.2$, py-2- $H), 8.69(1 \mathrm{H}, \mathrm{dd}, J=4.9$ and 1.2, py-6-H), $7.92(1 \mathrm{H}, \mathrm{d}, J=7.8$, py-4-H), $7.37(1 \mathrm{H}, \mathrm{dd}, J=7.8$ and 4.9, py-5-H), 4.69 $(1 \mathrm{H}, \mathrm{dd}, J=8.4$ and 5.0, $\mathrm{CH}), 3.79(3 \mathrm{H}, \mathrm{s}, \mathrm{CH}), 3.67-3.73\left(1 \mathrm{H}, \mathrm{m}, \mathrm{NC}^{\mathrm{d}} H_{a} \mathrm{H}_{\mathrm{b}}\right), 3.55-3.63(1 \mathrm{H}$, m, NC $\left.{ }^{\mathrm{d}} \mathrm{H}_{\mathrm{A}} H_{B}\right), 2.32-2.38\left(1 \mathrm{H}, \mathrm{m}, \mathrm{C}^{\mathrm{b}} H_{a} \mathrm{H}_{\mathrm{b}}\right), 2.02-2.13\left(2 \mathrm{H}, \mathrm{m}, \mathrm{C}^{\mathrm{b}} \mathrm{H}_{a} H_{b}\right.$ and $\left.\mathrm{C}^{\mathrm{g}} H_{a} \mathrm{H}_{\mathrm{b}}\right), 1.90-1.99$ $\left(1 \mathrm{H}, \mathrm{m}, \mathrm{C}^{\mathrm{g}} \mathrm{H}_{\mathrm{a}} H_{b}\right)$. Anal. Calcd for $\mathrm{C}_{12} \mathrm{H}_{14} \mathrm{~N}_{2} \mathrm{O}_{3}: \mathrm{C}, 61.53 ; \mathrm{H}, 6.02 ; \mathrm{N}, 11.96$. Found: $\mathrm{C}$, $61.70 ; \mathrm{H}, 6.05 ; \mathrm{N}, 11.88$.<smiles>COC(=O)[C@@H]1CCCN1C(=O)c1cccnc1</smiles>

Nic-Pro-OMe 
N-Nicotinylphenylglycine methyl ester (Nic-PhG-OMe). Yellow amorphous (2.90 g, 99\%): $[\alpha]_{\mathrm{D}}^{25}+90.0^{\circ}\left(c 0.52, \mathrm{CHCl}_{3}\right) ; \mathrm{IR}(\mathrm{KBr}) v_{\max } / \mathrm{cm}^{-1} 1745,1651(\mathrm{vC}=\mathrm{O}), 1215,1174(\mathrm{vC}-\mathrm{O}), 732$, $702(\gamma \mathrm{CH}) ;{ }^{1} \mathrm{H} \mathrm{NMR}\left(\mathrm{CDCl}_{3}, 400 \mathrm{MHz}\right) \delta 9.04(1 \mathrm{H}, \mathrm{d}, J=1.7, \mathrm{py}-2-H), 8.73(1 \mathrm{H}, \mathrm{dd}, J=4.8$ and 1.7, py-6- $H), 8.13(1 \mathrm{H}, \mathrm{dt}, J=7.8$ and 1.7, py-4- $H), 7.45-7.31\left(7 \mathrm{H}, \mathrm{m}, \mathrm{N} H, \mathrm{C}_{6} H_{5}\right.$, py-5- $\left.H\right)$, $5.77(1 \mathrm{H}, \mathrm{d}, J=6.8, \mathrm{CHCO}), 3.78\left(3 \mathrm{H}, \mathrm{s}, \mathrm{OCH}_{3}\right)$. Anal. Calcd for $\mathrm{C}_{15} \mathrm{H}_{14} \mathrm{~N}_{2} \mathrm{O}_{3} \bullet 0.2 \mathrm{CH}_{2} \mathrm{Cl}_{2}$ : C, 63.55; H, 5.05; N, 9.75. Found: C, 63.54; H, 5.03; N, 9.82.<smiles>COC(=O)[C@H](NC(=O)c1cccnc1)c1ccccc1</smiles>

Nic-PhG-OMe

Representative procedure for the synthesis of $N$-pyridiniuim iodides (Menshutkin Reaction): 3-[\{(1S)-1-Methoxycarbonylethyl\}carbamoyl]-1-methylpyridinium iodide (NAD ${ }^{+}$-Ala-OMe). A mixture of $N$-nicotinylalanine methyl ester (Nic-Ala-OMe) $(1.04 \mathrm{~g}$, $4.99 \mathrm{mmol})$ and iodomethane $\left(3.55 \mathrm{~g}, 25.0 \mathrm{mmol}\right.$ ) was heated at $70{ }^{\circ} \mathrm{C}$ in a tightly sealed glass reactor for $7 \mathrm{~h}$. After cooling to room temperature, the remaining iodomethane was removed under reduced pressure to give a crude product, which was then purified by colomun chromatography on silic gel with chloroform-methanol $(6: 1)$ as an eluent to afford the pure product as yellowish oil (1.66 g, 95\%): $[\alpha]^{21} \mathrm{D}-9.1\left(c 0.5, \mathrm{CH}_{3} \mathrm{OH}\right)$; IR $(\mathrm{KBr}) v_{\max } / \mathrm{cm}^{-1} 3242$ $(v \mathrm{NH}), 1739,1670(\mathrm{vC}=\mathrm{O}), 1212,1161(\mathrm{vC}-\mathrm{O}) ;{ }^{1} \mathrm{H}$ NMR (DMSO-d $\left.d_{6}, 400 \mathrm{MHz}\right) \delta 9.45(1 \mathrm{H}, \mathrm{d}$, $J=7.3, \mathrm{~N} H), 9.41(1 \mathrm{H}, \mathrm{s}, \mathrm{py}-2-H), 9.10(1 \mathrm{H}, \mathrm{d}, J=6.1$, py-6-H), $8.92(1 \mathrm{H}, \mathrm{d}, J=8.0$, py-4- $H)$, $8.22(1 \mathrm{H}, \mathrm{dd}, J=8.0$ and 6.1, py-5- $H), 4.54(1 \mathrm{H}$, quint, $J=7.3, \mathrm{CHCO}), 4.39\left(1 \mathrm{H}, \mathrm{s}, \mathrm{NCH}_{3}\right)$, $3.66\left(3 \mathrm{H}, \mathrm{s}, \mathrm{OCH}_{3}\right), 1.43\left(1 \mathrm{H}, \mathrm{d}, J=7.3, \mathrm{CHCH}_{3}\right)$. Anal. Calcd for $\mathrm{C}_{11} \mathrm{H}_{15} \mathrm{IN}_{2} \mathrm{O}_{3} \bullet$ bू: C, 35.89; H, 4.65; N, 7.61. Found: C, 35.84; H, 4.38; N, 7.49.

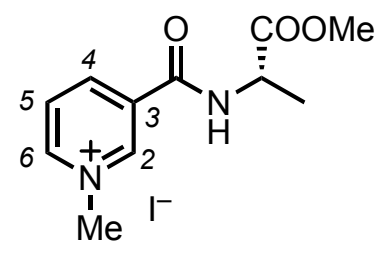

NAD ${ }^{+}$-Ala-OMe

\section{3-[\{(1S)-1-Methoxycarbonyl-2-methylpropyl\}carbamoyl]-1-methylpyridinium}

iodide

(NAD ${ }^{+}$-Val-OMe). $[\alpha]_{\mathrm{D}}^{25}-5.1^{\circ}\left(c 0.53, \mathrm{CHCl}_{3}\right)$; IR (KBr) $v_{\max } / \mathrm{cm}^{-1} 3255(v \mathrm{NH}), 1739,1671$ $(v \mathrm{C}=\mathrm{O}), 1210,1153(\mathrm{vC}-\mathrm{O}) ;{ }^{1} \mathrm{H} \mathrm{NMR}\left(\mathrm{CDCl}_{3}, 400 \mathrm{MHz}\right) \delta 9.83(1 \mathrm{H}, \mathrm{s}, \mathrm{py}-2-H), 9.23(1 \mathrm{H}, \mathrm{d}$, $J=5.9$, py-6-H), $8.96(1 \mathrm{H}, \mathrm{d}, J=8.1$, py-4-H), $8.45(1 \mathrm{H}, \mathrm{d}, J=7.6, \mathrm{~N} H), 8.17(1 \mathrm{H}, \mathrm{dd}, J=$ 8.1 and 5.9, pyr-5H), $4.65\left(3 \mathrm{H}, \mathrm{s}, \mathrm{NCH}_{3}\right), 4.52-4.55(1 \mathrm{H}, \mathrm{m}, \mathrm{NCHCO}), 3.76\left(3 \mathrm{H}, \mathrm{s}, \mathrm{OCH}_{3}\right)$, $2.46\left(1 \mathrm{H}\right.$, octet, $\left.J=6.8, \mathrm{C} H \mathrm{Me}_{2}\right), 1.14\left(3 \mathrm{H}, \mathrm{d}, J=6.8, \mathrm{CH}_{3}\right), 1.06\left(3 \mathrm{H}, \mathrm{d}, J=6.8, \mathrm{CH}_{3}\right)$. Anal. Calcd for $\mathrm{C}_{13} \mathrm{H}_{19} \mathrm{IN}_{2} \mathrm{O}_{3} \bullet 0.4 \mathrm{H}_{2} \mathrm{O}: \mathrm{C}, 40.51 ; \mathrm{H}, 5.18 ; \mathrm{N}, 7.27$. Found: $\mathrm{C}, 40.51 ; \mathrm{H}, 5.22 ; \mathrm{N}$, 7.05 . 


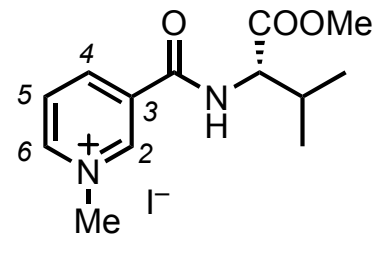

$\mathrm{NAD}^{+}-\mathrm{Val}-\mathrm{OMe}$

3-[\{(1S)-1-Methoxycarbonyl-2-phenylethyl $\}$ carbamoyl]-1-methylpyridinium

iodide

(NAD ${ }^{+}$-Phe-OMe). $[\alpha]_{\mathrm{D}}^{24}-50.3\left(c \quad 0.30, \mathrm{CHCl}_{3}\right) ; \mathrm{IR}(\mathrm{KBr}) v_{\max } / \mathrm{cm}^{-1} 3237(\mathrm{vNH}), 1740$, $1673(v \mathrm{C}=\mathrm{O}), 1209,1097(\mathrm{vC}-\mathrm{O}), 753,704,670(\gamma \mathrm{CH}) ;{ }^{1} \mathrm{H}$ NMR (DMSO- $\left.d_{6}, 400 \mathrm{MHz}\right) \delta 9.55$ $(1 \mathrm{H}, \mathrm{d}, J=7.8, \mathrm{~N} H), 9.32(1 \mathrm{H}, \mathrm{s}, \mathrm{py}-2-H), 9.08(1 \mathrm{H}, \mathrm{d}, J=6.1, \mathrm{py}-6-H), 8.81(1 \mathrm{H}, \mathrm{d}, J=8.0$, py-4- $H), 8.22$ (1 H, dd, $J=8.0$ and 6.1, py-5-H), 7.25-7.28 (4 H, m, Ph-2', 3', 5',6'-H), 7.12-7.23 $\left(1 \mathrm{H}, \mathrm{m}, \mathrm{Ph}-4^{\prime}-H\right), 4.76\left(1 \mathrm{H}, \mathrm{ddd}, J=10.0,7.8\right.$, and 5.4, NCHCO), $4.36\left(3 \mathrm{H}, \mathrm{s}, \mathrm{NCH}_{3}\right), 3.64$ $\left(3 \mathrm{H}, \mathrm{s}, \mathrm{OCH}_{3}\right), 3.21\left(1 \mathrm{H}, \mathrm{dd}, J=13.9\right.$ and 5.4, $\left.\mathrm{CH}_{A} \mathrm{H}_{\mathrm{B}} \mathrm{Ph}\right), 3.08(1 \mathrm{H}, \mathrm{dd}, J=13.9$ and 5.4, $\mathrm{CH}_{\mathrm{A}} H_{B} \mathrm{Ph}$ ). Anal. Calcd for $\mathrm{C}_{17} \mathrm{H}_{19} \mathrm{IN}_{2} \mathrm{O}_{3} \bullet 0.8 \mathrm{H}_{2} \mathrm{O}: \mathrm{C}, 46.34 ; \mathrm{H}, 4.71 ; \mathrm{N}, 6.36$. Found: $\mathrm{C}$, $46.51 ; \mathrm{H}, 4.70 ; \mathrm{N}, 6.19$.

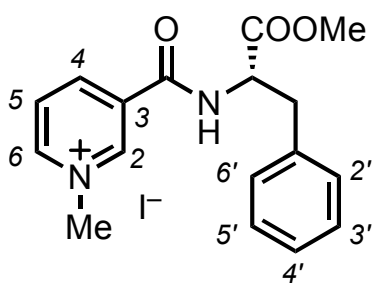

NAD ${ }^{+}$-Phe-OMe

3-[\{(2S)-2-Methoxycarbonylpyrolidinyl\}carbonyl]-1-methylpyridinium

iodide (NAD ${ }^{+}$-Pro-OMe). $[\alpha]^{25}{ }_{\mathrm{D}}-65.1^{\circ}\left(c 0.23, \mathrm{CH}_{3} \mathrm{OH}\right)$; IR $(\mathrm{KBr}) v_{\max } / \mathrm{cm}^{-1} 1736,1648(\mathrm{vC}=\mathrm{O})$, 1216, $1179(\mathrm{vC}-\mathrm{O}) ;{ }^{1} \mathrm{H}$ NMR (DMSO- $\left.d_{6}, 400 \mathrm{MHz}\right) \delta 9.26(1 \mathrm{H}, \mathrm{s}, \mathrm{py}-2-H), 9.06(1 \mathrm{H}, \mathrm{d}, J=$ 6.1, py-6-H), $8.71(1 \mathrm{H}, \mathrm{d}, J=8.1$, py-4-H), 8.19 (1 H, dd, $J=8.1$ and 6.1, py-5- $H), 4.54-4.51$ $\left(1 \mathrm{H}, \mathrm{m}, \mathrm{NC}^{\mathrm{a}} H\right), 4.37\left(3 \mathrm{H}, \mathrm{s}, \mathrm{NCH}_{3}\right), 3.68\left(3 \mathrm{H}, \mathrm{s}, \mathrm{OCH}_{3}\right), 3.62-3.59\left(2 \mathrm{H}, \mathrm{m}, \mathrm{NC}^{\mathrm{d}} H_{2}\right), 2.35-2.26$ $\left(1 \mathrm{H}, \quad \mathrm{m}, \quad \mathrm{C}^{\mathrm{b}} \mathrm{H}_{a} \mathrm{H}_{\mathrm{b}}\right), \quad 1.98-1.89 \quad\left(3 \mathrm{H}, \quad \mathrm{m}, \mathrm{C}^{\mathrm{b}} \mathrm{H}_{\mathrm{a}} H_{b}\right.$ and $\left.\mathrm{C}^{\mathrm{g}} \mathrm{H}_{2}\right)$. Anal. Calcd for $\mathrm{C}_{13} \mathrm{H}_{17} \mathrm{IN}_{2} \mathrm{O}_{3} \bullet 0.6 \mathrm{H}_{2} \mathrm{O}: \mathrm{C}, 40.35 ; \mathrm{H}, 4.74 ; \mathrm{N}, 7.24$. Found: $\mathrm{C}, 40.24 ; \mathrm{H}, 4.61 ; \mathrm{N}, 6.97$.<smiles>CO[N+](=O)[O-]</smiles>

3-[\{(1S)-1-Methoxycarbonyl-1-phenylmethyl\}carbonyl]-1-methylpyridinium iodide (NAD ${ }^{+}$-PhG-OMe). $[\alpha]^{25}{ }_{\mathrm{D}}+50.6^{\circ}\left(c \quad 0.30, \mathrm{CHCl}_{3}\right)$; IR $(\mathrm{KBr}) v_{\max } / \mathrm{cm}^{-1} 3236(\mathrm{vNH}), 1742$, $1670(\mathrm{vC}=\mathrm{O}), 1211,1103(\mathrm{vC}-\mathrm{O}), 746,702,699(\gamma \mathrm{CH}) ;{ }^{1} \mathrm{H}$ NMR $\left(\mathrm{CDCl}_{3}, 400 \mathrm{MHz}\right) \delta 9.75$ $(1 \mathrm{H}, \mathrm{s}, \mathrm{py}-2-H), 9.11(1 \mathrm{H}, \mathrm{d}, J=5.9$, py-6-H), $9.01(1 \mathrm{H}, \mathrm{d}, J=7.3, \mathrm{~N} H), 8.87(1 \mathrm{H}, \mathrm{d}, J=8.1$, 
py-4- $H$ ), 8.08 (1H, dd, $J=8.1$ and 5.9, py-5- $H), 7.60$ (2 H, d, $J=7.3$, Ph-2',6'- $H), 7.31-7.39$ (3H, m, Ph-3',4',5'-H), 5.75 (1H, d, $J=7.3, \mathrm{NCHCO}), 4.54$ (3H, s, $\left.\mathrm{NCH}_{3}\right), 3.72\left(3 \mathrm{H}, \mathrm{s}, \mathrm{OCH}_{3}\right)$. Anal. Calcd for $\mathrm{C}_{16} \mathrm{H}_{17} \mathrm{IN}_{2} \mathrm{O}_{3} \bullet 0.6 \mathrm{H}_{2} \mathrm{O}: \mathrm{C}, 45.43 ; \mathrm{H}, 4.34 ; \mathrm{N}, 6.62$. Found: $\mathrm{C}, 45.45 ; \mathrm{H}, 4.34 ; \mathrm{N}$, 6.75 .<smiles>COC(=O)[C@H](NC(=O)c1ccc[n+](C)c1)c1ccccc1</smiles>

$\mathrm{NAD}+\mathrm{PhG}-\mathrm{OMe}$

Representative procedure for the synthesis of 1,4-dihydropyridines: (S)-Methyl 2-(1,4-dihydro-1-methylpyridine-3-carboxamido)propanoate (1a). To an argon-purged solution of sodium dithionite ( $4.3 \mathrm{~g}, 25 \mathrm{mmol})$ in $5 \%$ aqueous $\mathrm{NaHCO}_{3}(16.0 \mathrm{~mL})$ was added in one portion a mixture of 3-[\{(1S)-1-methoxycarbonylethyl $\}$ carbamoyl]-1-methylpyridinium iodide $\left(\mathrm{NAD}^{+}\right.$-Ala-OMe) $(983 \mathrm{mg}, 2.81 \mathrm{mmol})$ in dichloromethane $(16.0 \mathrm{~mL})$, which was purged with argon prior to the addition, under argon atmosphere at room temperature. The mixture was then vigorously stirred at room temperature for $3 \mathrm{~h}$ in the dark under argon. To the reaction mixture were added water $(30 \mathrm{~mL})$ and dichloromethane $(30 \mathrm{~mL})$, and the organic phase was separated. The organic layer was further washed with water $(20 \mathrm{~mL} \mathrm{x} 2)$ and then dried over sodium sulfate. After evaporation of the solvent, the pure product was obtained as yellow amorphous solid (363 mg, 57\%): $[\alpha]^{25}{ }_{\mathrm{D}}+25.8\left(c 0.16, \mathrm{CHCl}_{3}\right)$; IR $(\mathrm{KBr}) v_{\max } / \mathrm{cm}^{-1} 3397$ (vNH), 1739, $1682(v \mathrm{C}=\mathrm{O}), 1206,1099(v \mathrm{C}-\mathrm{O}) ;{ }^{1} \mathrm{H}$ NMR $\left(\mathrm{CDCl}_{3}, 400 \mathrm{MHz}\right) \delta 6.99(1 \mathrm{H}, \mathrm{s}$, py-2- $H$ ), 5.70-5.68 (2 H, m, py-6- $H$ and $\mathrm{N} H), 4.74-4.67(2 \mathrm{H}, \mathrm{m}$, py-5- $H$ and $\mathrm{NHCH}), 3.75(3 \mathrm{H}$, s, $\left.\mathrm{OCH}_{3}\right), 3.18-3.15(2 \mathrm{H}, \mathrm{m}, \mathrm{py}-4-\mathrm{H} \times 2), 2.93\left(3 \mathrm{H}, \mathrm{s}, \mathrm{NCH}_{3}\right), 1.42\left(3 \mathrm{H}, \mathrm{d}, J=7.3, \mathrm{CHCH}_{3}\right)$; ${ }^{13} \mathrm{C}$ NMR (400 MHz, $\left.\mathrm{CDCl}_{3}\right) \delta 174.2(\mathrm{CONH}), 167.4$ (COO), 139.9 (py-2-C), 129.9 (py-6-C), 102.3 (py-3-C), 98.2 (py-5-C), $52.4\left(\mathrm{OCH}_{3}\right), 47.9(\mathrm{CH}), 40.7\left(\mathrm{NCH}_{3}\right), 22.1$ (py-4-C), 18.8 $\left(\mathrm{CH}_{3}\right)$; UV (in $\left.\mathrm{CHCl}_{3}\right) \lambda_{\max } / \mathrm{nm}\left(\varepsilon / \mathrm{M}^{-1} \mathrm{~cm}^{-1}\right) 353$ (2010), 273 (4920). Anal Calcd for $\mathrm{C}_{11} \mathrm{H}_{16} \mathrm{~N}_{2} \mathrm{O}_{3} \cdot 0.2 \mathrm{CH}_{2} \mathrm{Cl}_{2}$ : C, 55.76; H, 6.85; N, 11.61. Found: C, 55.66; H, 7.04; N, 11.62.

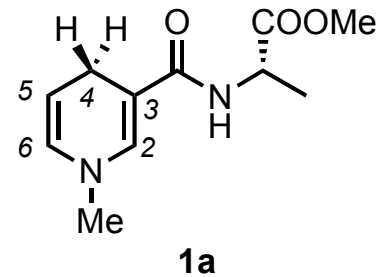

(S)-Methyl 2-(1,4-dihydro-1-methylpyridine-3-carboxamido)-3-methylbutanoate (1b). $[\alpha]_{\mathrm{D}}^{25}+18.7\left(c 0.11, \mathrm{CHCl}_{3}\right)$; IR $(\mathrm{KBr}) v_{\max } / \mathrm{cm}^{-1} 3298(v \mathrm{NH}), 1739,1683(\mathrm{vC}=\mathrm{O}), 1198,1090$ (vC-O); ${ }^{1} \mathrm{H}$ NMR $\left(400 \mathrm{MHz}, \mathrm{CDCl}_{3}\right) \delta 6.99(1 \mathrm{H}, \mathrm{d}, J=1.2$, py-2- $H) .5 .69(1 \mathrm{H}, \mathrm{dd}, J=8.1$ and 1.2, py-6-H), $5.61(1 \mathrm{H}, \mathrm{d}, J=8.0, \mathrm{~N} H), 4.73(1 \mathrm{H}, \mathrm{dd}, J=8.1$ and 3.4, py-5-H), $4.67(1 \mathrm{H}, \mathrm{dd}, J$ $=8.0$ and 5.0, NHCHCO), 3.15-3.25 (2 H, m, py-4-H $x 2), 2.93(3 \mathrm{H}, \mathrm{s}, \mathrm{NCH}), 2.11-2.22(1 \mathrm{H}$, 
m, $\left.C H \mathrm{Me}_{2}\right), 0.94\left(3 \mathrm{H}, \mathrm{d}, J=7.0, \mathrm{CH}_{3}\right), 0.91\left(3 \mathrm{H}, \mathrm{d}, J=7.0, \mathrm{CH}_{3}\right) ;{ }^{13} \mathrm{C} \mathrm{NMR}(400 \mathrm{MHz}$, $\left.\mathrm{CDCl}_{3}\right) \delta 173.2(C \mathrm{ONH}), 167.8(C \mathrm{OO}), 139.9$ (py-2-C), 130.0 (py-6-C), 102.2 (py-3-C), 98.4 (py-5-C), $56.8(\mathrm{CH}), 52.1\left(\mathrm{OCH}_{3}\right), 40.7\left(\mathrm{NCH}_{3}\right), 31.5(\mathrm{CH}), 22.2$ (py-4-C), $19.0\left(\mathrm{CH}_{3}\right), 17.9$ $\left(\mathrm{CH}_{3}\right)$; UV (in $\left.\mathrm{CHCl}_{3}\right) \lambda_{\max } / \mathrm{nm}\left(\varepsilon / \mathrm{M}^{-1} \mathrm{~cm}^{-1}\right) 356$ (2830), 282 (4440). Anal. Calcd for $\mathrm{C}_{13} \mathrm{H}_{20} \mathrm{~N}_{2} \mathrm{O}_{3} \bullet 0.8 \mathrm{H}_{2} \mathrm{O}$ : C, 58.54; H, 8.16; N, 10.50. Found: C, 58.54; H, 8.27; N, 10.20.<smiles>[CH][C@@H]1C=CN(C)C=C1C(=O)N[C@@H](C(C)=O)C(C)C</smiles>

(S)-Methyl 2-(1,4-dihydro-1-methylpyridine-3-carboxamido)-3-phenylpropanoate (1c). $[\alpha]_{\mathrm{D}}^{25}+38.1\left(c 0.11, \mathrm{CHCl}_{3}\right)$; IR (KBr) $v_{\max } / \mathrm{cm}^{-1} 3333(v \mathrm{NH}), 1740,1682(\mathrm{vC}=\mathrm{O}), 1204,1104$ (vC-O), 746, $702(\gamma \mathrm{CH}) ;{ }^{1} \mathrm{H} \mathrm{NMR}\left(\mathrm{CDCl}_{3}, 400 \mathrm{MHz}\right) \delta 7.21-7.30\left(5 \mathrm{H}, \mathrm{m}, \mathrm{C}_{6} H_{5}\right), 6.99(1 \mathrm{H}, \mathrm{s}$, py-2- $H$ ), 5.67 ( $1 \mathrm{H}, \mathrm{d}, J=8.0$, py-6- $H), 5.55$ (1 H, d, $J=7.8, \mathrm{~N} H), 4.95-5.01$ (1 H, m, NHCHCO), 4.69 (1 H, dt, $J=7.8$ and 3.4, py-5-H), $3.72\left(3 \mathrm{H}, \mathrm{s}, \mathrm{OCH}_{3}\right), 3.10-3.19(2 \mathrm{H}, \mathrm{m}$, $\mathrm{CH}_{2} \mathrm{Ph}$ ), 2.98-3.10 (2 H, m, py-4- $\mathrm{H}$ x 2), 2.93 (3 H, s, NCH$) ;{ }^{13} \mathrm{C}-\mathrm{NMR}\left(400 \mathrm{MHz}, \mathrm{CDCl}_{3}\right)$ $\delta 172.5$ (CONH), 167.3 (COO), 139.9 (py-2-C), 136.1 (Ph-1'-C), 129.9 (py-6-C), 129.3 (Ph-3',5'-C), 128.5 (Ph-2',6'-C), 127.0 (Ph-4'-C), 102.4 (py-3-C), 98.1 (py-5-C), $53.0(\mathrm{NHCH})$, $52.2\left(\mathrm{OCH}_{3}\right), 40.7\left(\mathrm{NCH}_{3}\right), 38.0\left(\mathrm{PhCH}_{2}\right), 21.9(4-C)$; UV (in $\left.\mathrm{CHCl}_{3}\right) \lambda_{\max } / \mathrm{nm}\left(\varepsilon / \mathrm{M}^{-1} \mathrm{~cm}^{-1}\right) 358$ (3850), 282 (4600). Anal. Calcd for $\mathrm{C}_{17} \mathrm{H}_{20} \mathrm{~N}_{2} \mathrm{O}_{3} \bullet 0.5 \mathrm{H}_{2} \mathrm{O}: \mathrm{C}, 66.00 ; \mathrm{H}, 6.84 ; \mathrm{N}, 9.06$. Found: C, 65.87; H, 6.71; N, 9.12.<smiles></smiles>

1,4-Dihydro-3-[(2S)-(2-methoxycarbonylpyrolidinyl)carbonyl]-1-methylpyridine

(1d). $[\alpha]^{25}{ }_{\mathrm{D}}-1.88^{\circ}\left(c=0.12, \mathrm{CHCl}_{3}\right) ;{ }^{1} \mathrm{H}-\mathrm{NMR}\left(400 \mathrm{MHz}, \mathrm{CDCl}_{3}\right) \delta 6.47(1 \mathrm{H}, \mathrm{s}, 2-H), 5.65(1 \mathrm{H}, \mathrm{d}$, $J=7.6$, py-6- $H$ ), 4.64 ( $1 \mathrm{H}, \mathrm{dt}, J=7.6$ and 3.5, py-5- $H), 4.53$ ( $1 \mathrm{H}, \mathrm{d}, J=7.6, \mathrm{NCHCO}), 3.73$ $\left(3 \mathrm{H}, \mathrm{s}, \mathrm{OCH}_{3}\right), 3.63-3.73\left(2 \mathrm{H}, \mathrm{m}, \mathrm{NC}^{\mathrm{d}} H_{2}\right), 3.23(1 \mathrm{H}, \mathrm{dd}, J=18.4$ and 3.5, py-4- $H), 3.06(1 \mathrm{H}$, $\mathrm{dd}, J=18.4$ and 3.5, py-4-H), $2.89(3 \mathrm{H}, \mathrm{s}, \mathrm{NCH}), 2.18-2.26\left(1 \mathrm{H}, \mathrm{m}, \mathrm{C}^{\mathrm{b}} H_{a} \mathrm{H}_{\mathrm{b}}\right), 1.99-2.07(1 \mathrm{H}$, m, $\left.\mathrm{C}^{\mathrm{b}} \mathrm{H}_{\mathrm{a}} H_{b}\right), 1.84-1.94\left(2 \mathrm{H}, \mathrm{m}, \mathrm{C}^{\mathrm{g}} H_{2}\right) ;{ }^{13} \mathrm{C}-\mathrm{NMR}\left(400 \mathrm{MHz}, \mathrm{CDCl}_{3}\right) \delta 173.6(\mathrm{CONH}), 170.1$ (COO), 138.9 (py-2-C), 129.8 (py-6-C), 102.2 (py-5-C), 101.3 (py-3-C), $59.9\left(C^{a} \mathrm{H}\right), 52.1$ $\left(\mathrm{OCH}_{3}\right), 49.2\left(C^{d} \mathrm{H}_{2}\right), 40.7\left(\mathrm{NCH}_{3}\right), 29.2\left(C^{g} \mathrm{H}_{2}\right), 25.4\left(C^{b} \mathrm{H}_{2}\right), 23.0$ (py-4-C); UV (in $\left.\mathrm{CHCl}_{3}\right)$ $\lambda_{\max } / \mathrm{nm}\left(\varepsilon / \mathrm{M}^{-1} \mathrm{~cm}^{-1}\right) 351$ (2990), 284 (4470). Anal. Calcd for $\mathrm{C}_{13} \mathrm{H}_{18} \mathrm{~N}_{2} \mathrm{O}_{3} \cdot 0.5 \mathrm{H}_{2} \mathrm{O}: \mathrm{C}, 60.22$; H, 7.39; N, 10.80. Found: C, 60.33; H, 7.20; N, 10.78 . 
<smiles></smiles>

(S)-methyl 2-(1,4-dihydro-1-methylpyridine-3-carboxamido)-2-phenylacetate (1e). $[\alpha]^{25}$ +7.8 $\left(c 0.10, \mathrm{CHCl}_{3}\right) ;{ }^{1} \mathrm{H}-\mathrm{NMR}\left(400 \mathrm{MHz}, \mathrm{CDCl}_{3}\right) \delta 7.28-7.39\left(5 \mathrm{H}, \mathrm{m}, \mathrm{C}_{6} H_{5}\right), 6.98(1 \mathrm{H}, \mathrm{s}$, py-2-H), $6.12(1 \mathrm{H}, \mathrm{d}, J=6.3 \mathrm{~Hz}, \mathrm{~N} H), 5.67(2 \mathrm{H}, \mathrm{m}, \mathrm{NHCH}$ and py-6-H), 4.70-4.74 $(1 \mathrm{H}, \mathrm{m}$, py-5-H), $3.72\left(3 \mathrm{H}, \mathrm{s}, \mathrm{OCH}_{3}\right), 3.19\left(2 \mathrm{H}, \mathrm{ABq}, J=16.8 \mathrm{~Hz}\right.$, py-4-Hx2), $2.90\left(3 \mathrm{H}, \mathrm{s}, \mathrm{NCH}_{3}\right)$; ${ }^{13} \mathrm{C}-\mathrm{NMR}\left(400 \mathrm{MHz}, \mathrm{CDCl}_{3}\right) \delta 171.8(\mathrm{CONH}), 167.2$ (COO), 140.0 (py-2-C), 137.0 (Ph-1'-C), 129.8 (py-6-C), 128.8 (Ph-2',6'-C), 128.2 (Ph-4'-C), 127.2 (Ph-3',5'-C), 102.4 (py-5-C), 97.9 (py-3-C), $56.3(\mathrm{CH}), 52.6\left(\mathrm{O} \mathrm{CH}_{3}\right), 40.6\left(\mathrm{NCH}_{3}\right), 22.0$ (py-4-C); IR $(\mathrm{KBr}) v_{\max } / \mathrm{cm}^{-1} 3377$ $(v \mathrm{NH}), 1739,1686(\mathrm{vC}=\mathrm{O}), 1193,1107(\mathrm{vC}-\mathrm{O}), 730,688(\gamma \mathrm{CH}) ; \mathrm{UV}$ (in $\left.\mathrm{CHCl}_{3}\right) \lambda_{\max } / \mathrm{nm}$ $\left(\varepsilon / \mathrm{M}^{-1} \mathrm{~cm}^{-1}\right) 358$ (3670), 282 (4390). Anal. Calcd for $\mathrm{C}_{16} \mathrm{H}_{18} \mathrm{~N}_{2} \mathrm{O}_{3} \cdot 0.5 \mathrm{H}_{2} \mathrm{O}: \mathrm{C}, 65.07 ; \mathrm{H}, 6.48$; N, 9.49. Found: C, 64.88; H, 6.33; N, 9.57.

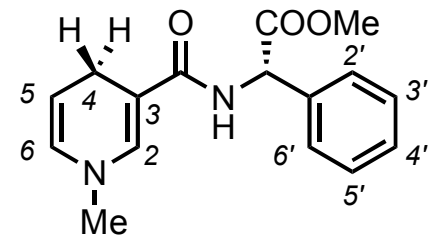

$1 e$

Asymmetric Reductions. Since strict regulation of the water content in the reaction system is important to obtain reliable and reproducible data, ${ }^{2}$ all the reagents, the NADH models (1a-e) and magnesium perchlorate, were dried over $\mathrm{P}_{2} \mathrm{O}_{5}$ in vacuo prior to use, and the asymmetric reduction of methyl benzoylformate $(0.2 \mathrm{mmol})$ with $1(0.2 \mathrm{mmol})$ was performed in dehydrated acetonitrile $(4.0 \mathrm{~mL})$ under argon atmosphere in the presence of anhydrous magnesium perchlorate $(0.2 \mathrm{mmol})^{2,3}$ for 72 hours at given temperatures ranging from -35 to $72{ }^{\circ} \mathrm{C}$ in the dark. After terminating the reaction by adding water to the reaction mixture, the solvents were removed under reduced pressure. Diethyl ether and water were added to the residue, and then the organic layer was separated and dried over anhydrous sodium sulfate. After evaporation of the solvent, the residue was dissolved in acetonitrile $(10 \mathrm{~mL})$. The chemical yield for each run was determined by analyzing the acotonitrile solution on a gas chromatography (GC), ${ }^{3}$ which demonstrated that only the reduction of the ketoester proceeded to give the chiral methyl mandelate in moderate to excellent chemical yields. The enantiomeric excess (ee) of the produced methyl mandelate was determined by HPLC analysis ${ }^{3}$ after purifying the product by a preparative thin-layer chromatography. Control experiments demonstrated that no racemization of the chiral methyl manderate occurred even at the highest temperature employed. 


\section{References.}

1. Endo, T.; Hayashi, Y.; Okawara, M. Chem. Lett. 1977, 6, 391-394.

2. Okamura, M.; Mikata, Y.; Yamazaki, N.; Tsutsumi, A.; Ohno, A. Bull. Chem. Soc. Jpn. 1993, 66, 1191-1196.

3. Katoh, A.; Naruse, S.; Ohkanda, J.; Yamamto, H. Heterocycles 1997, 45, 1441-1446. 


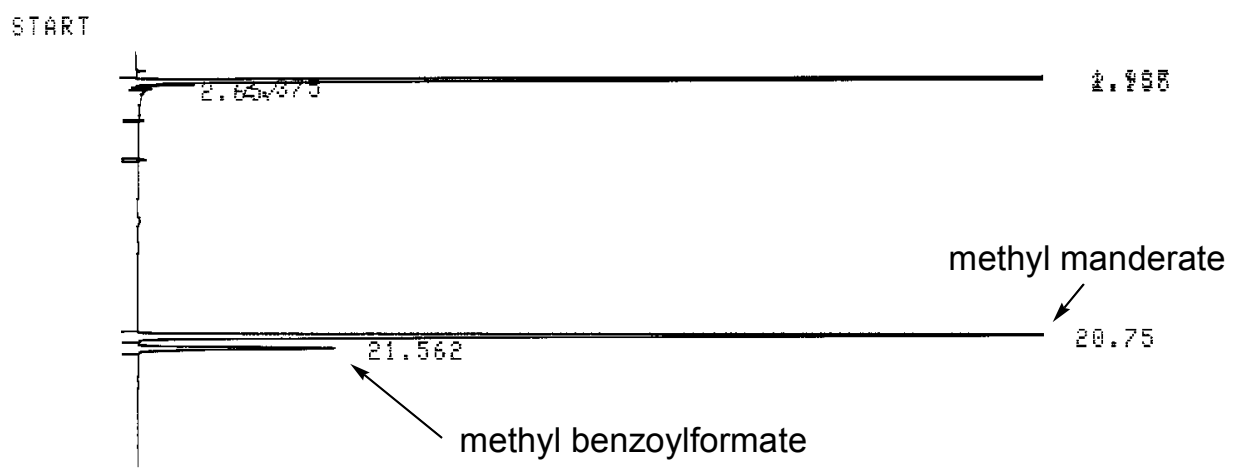

Figure S1. A selected GC chromatogram for the reduction of methyl benzoylformate with 1e at $0{ }^{\circ} \mathrm{C}$, analyzed on a $\mathrm{J} \& \mathrm{~W}$ Scientific DB-5MS capillary column $(0.32 \mathrm{~mm}$ I.D. $\mathrm{x} 30 \mathrm{~m})$ under the following conditions: carrier gas, nitrogen; column temperature, $110^{\circ} \mathrm{C}$; injector/detector temperature, $250{ }^{\circ} \mathrm{C}$.

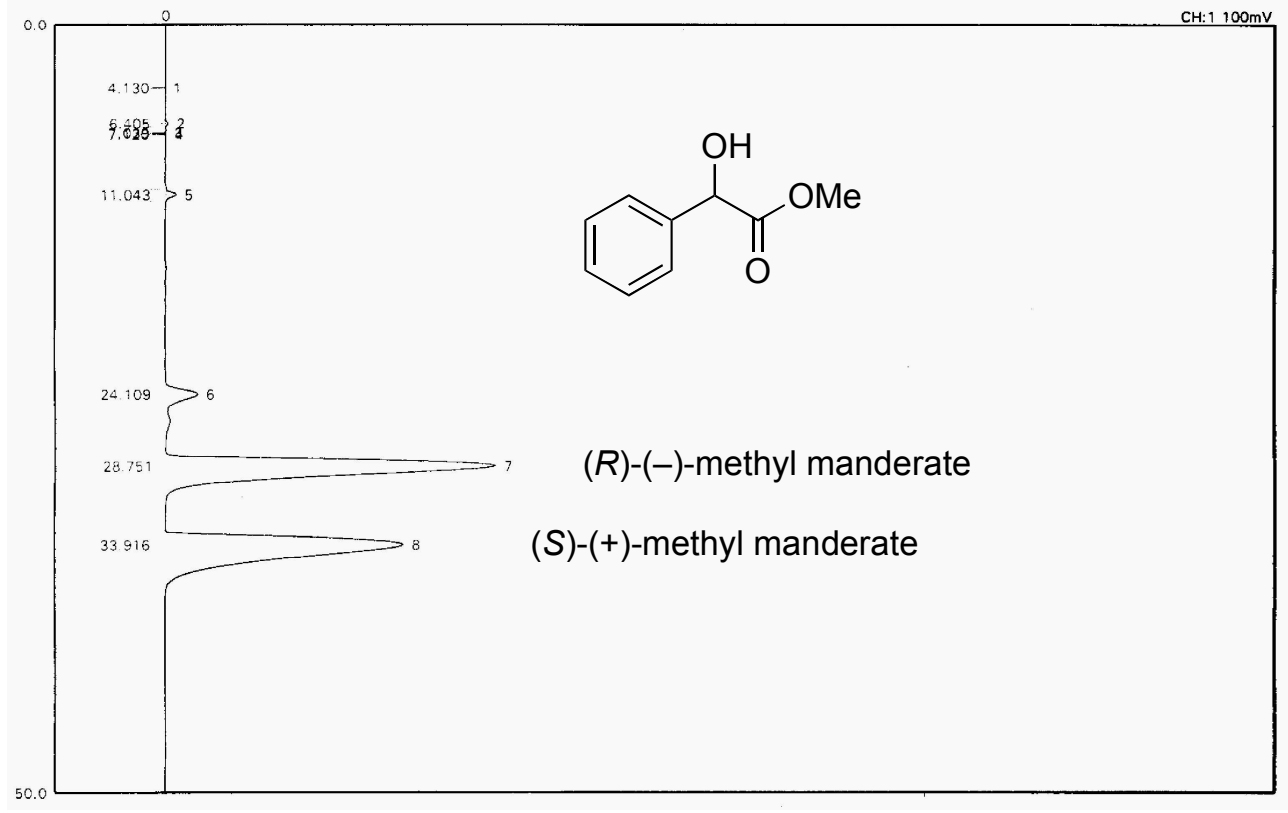

Figure S2. A typical HPLC chromatogram for rascemic methyl manderate analyzed on a Daicel Chiralcel OJ column (4.6 mm I.D. x $250 \mathrm{~nm}$ ) with hexane:2-propanol (9:1) as an eluent at $0.5 \mathrm{ml} / \mathrm{min}$ flow rate. 

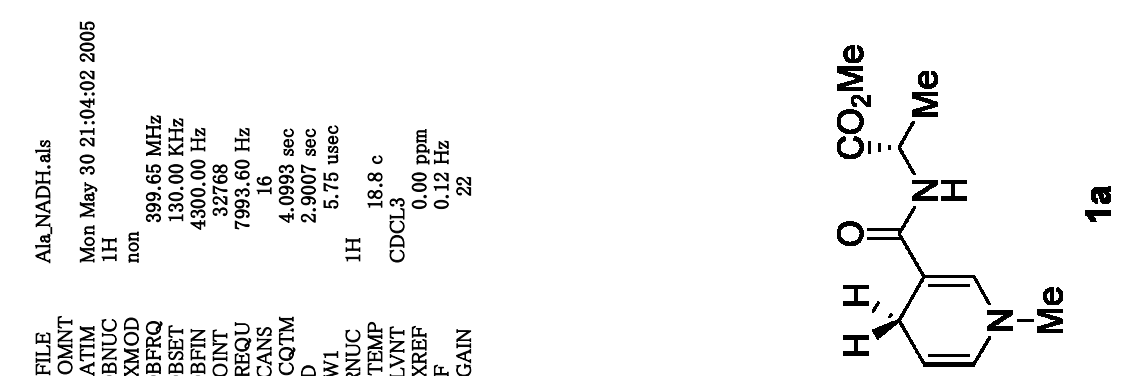

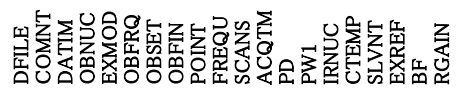

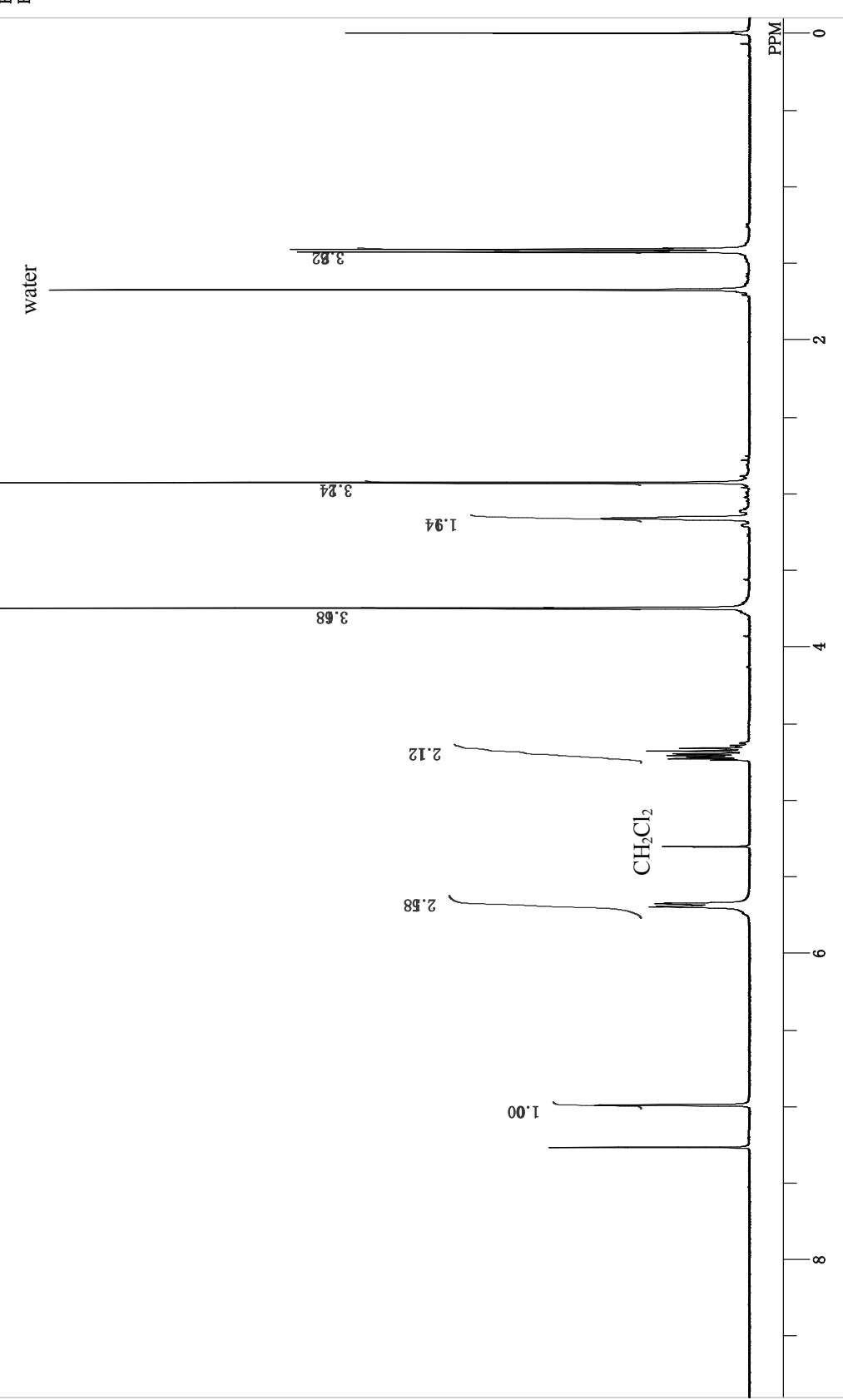



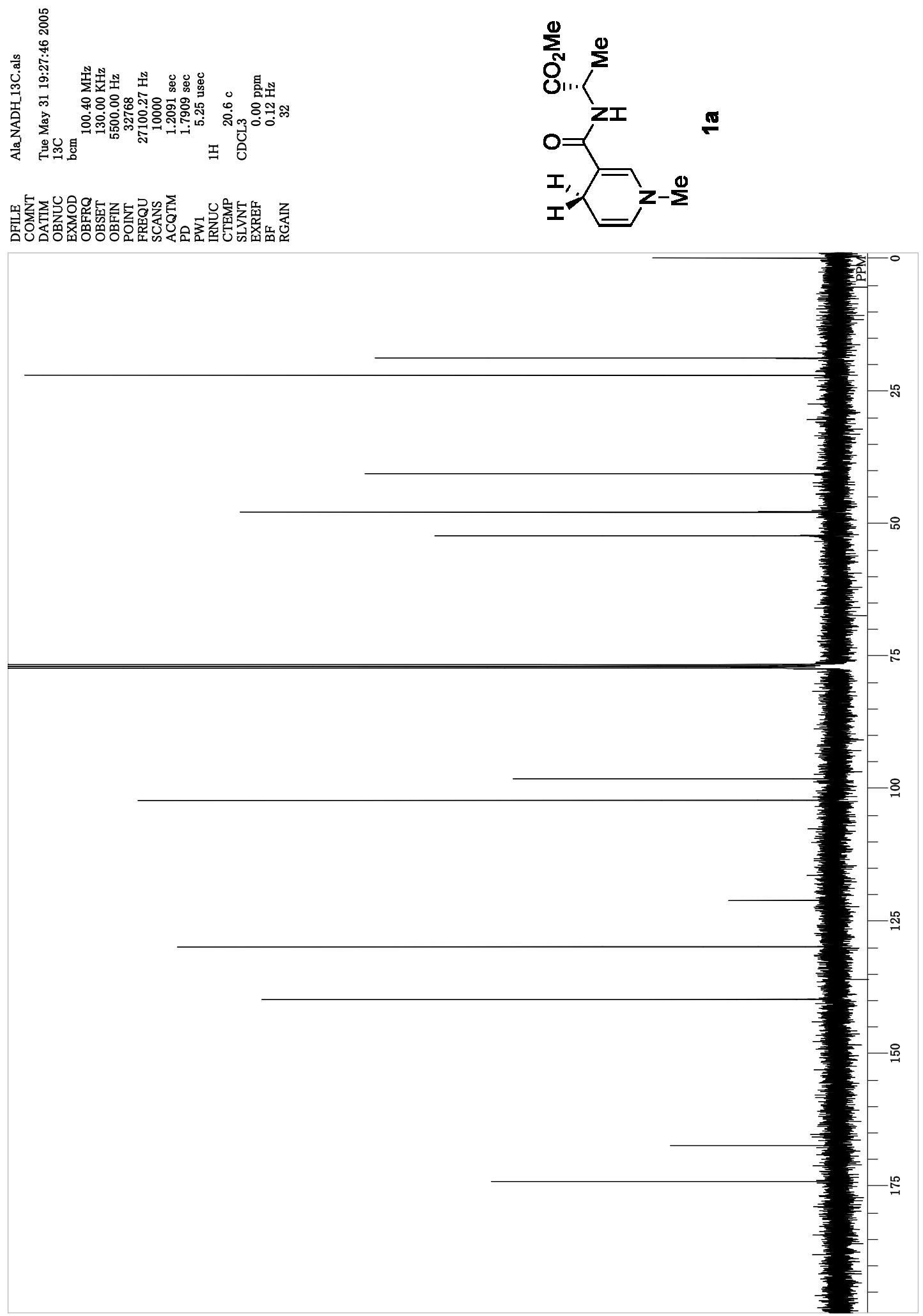

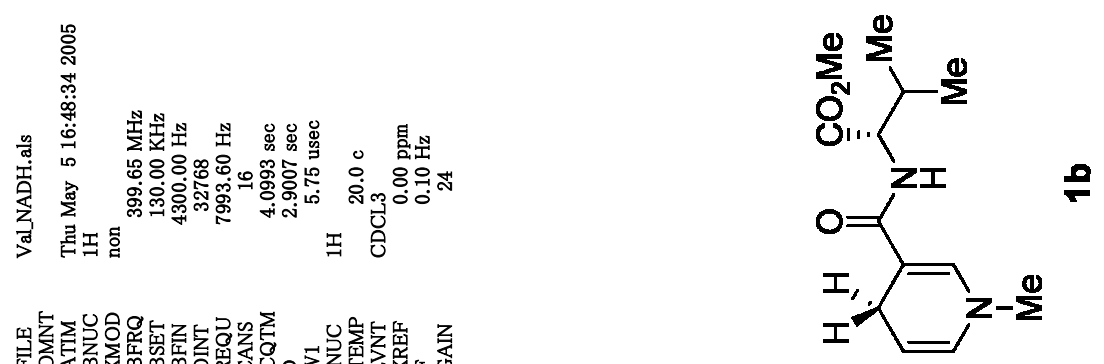

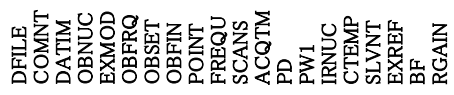

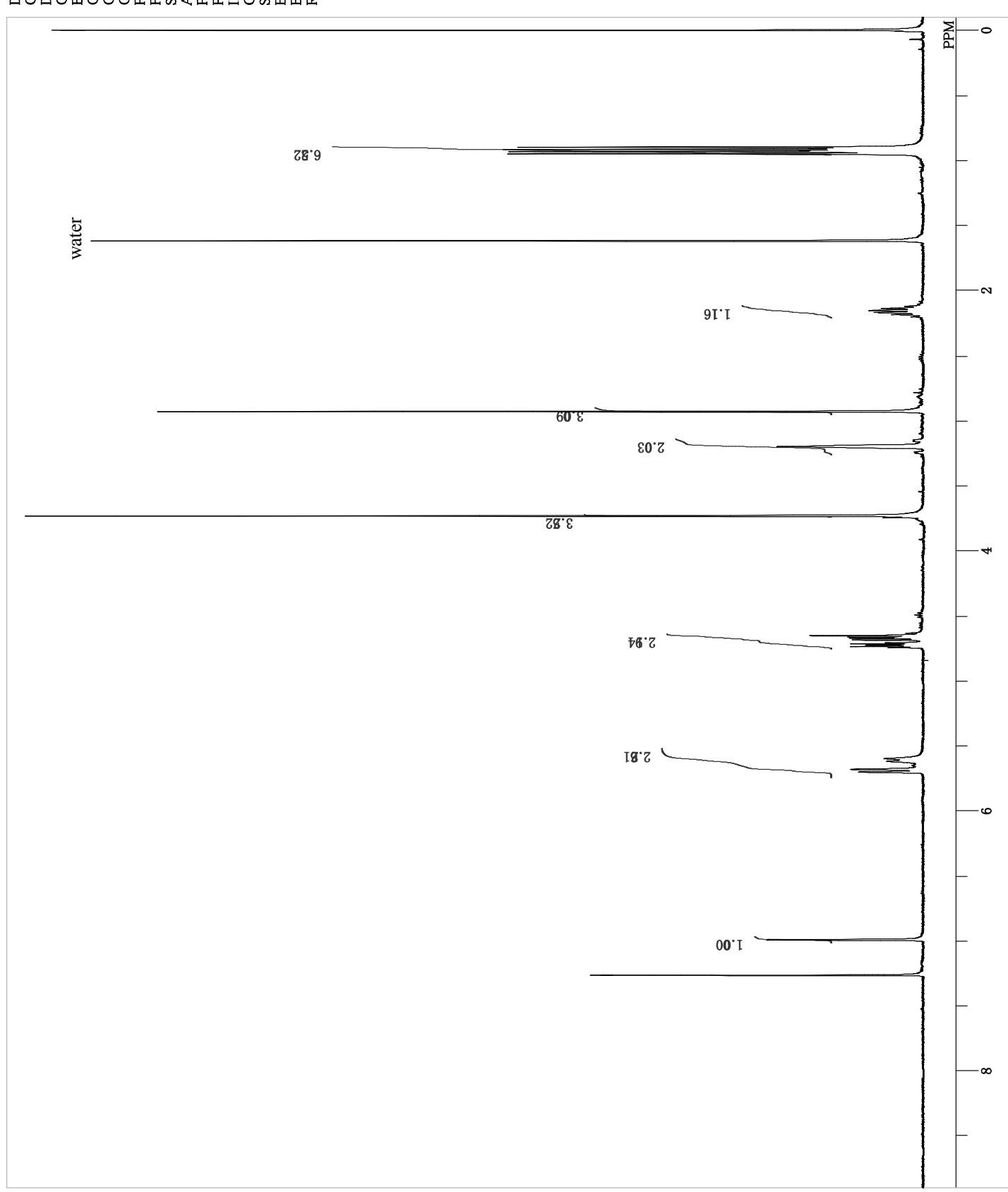



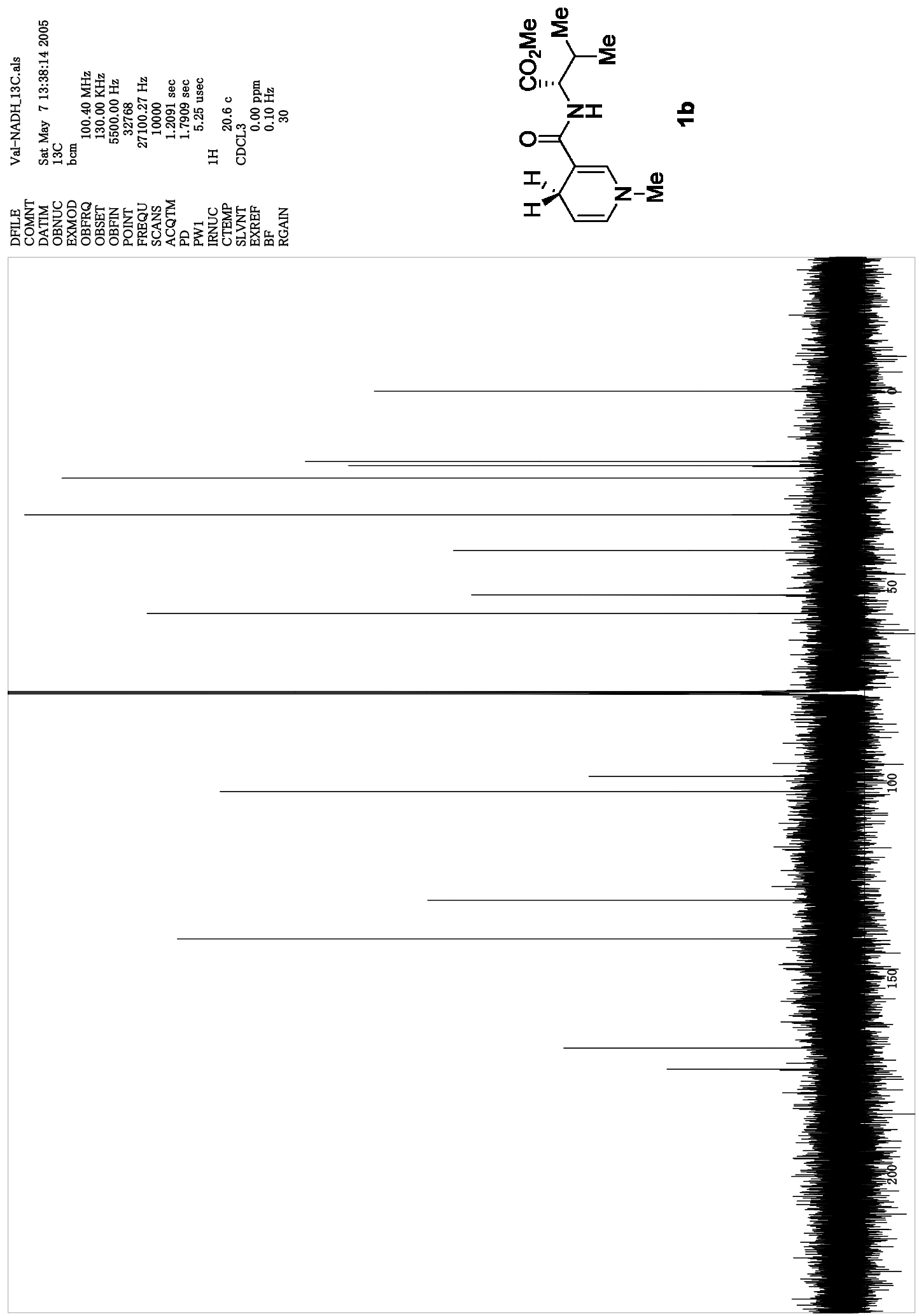

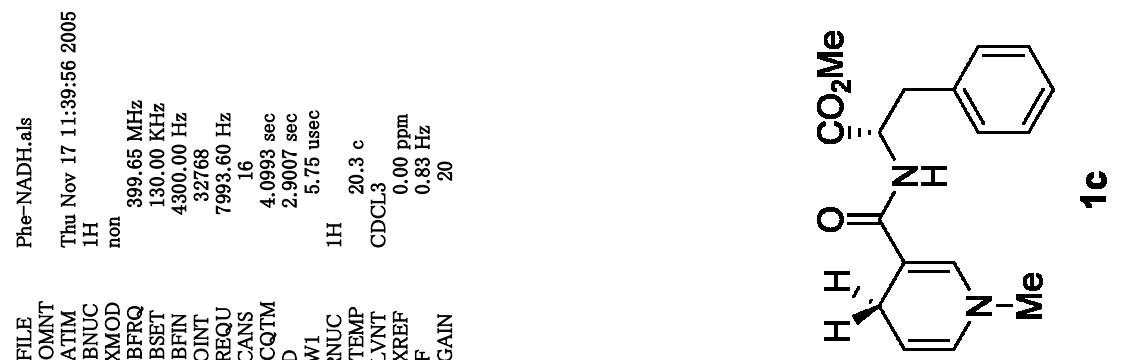

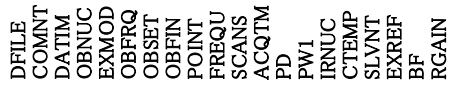

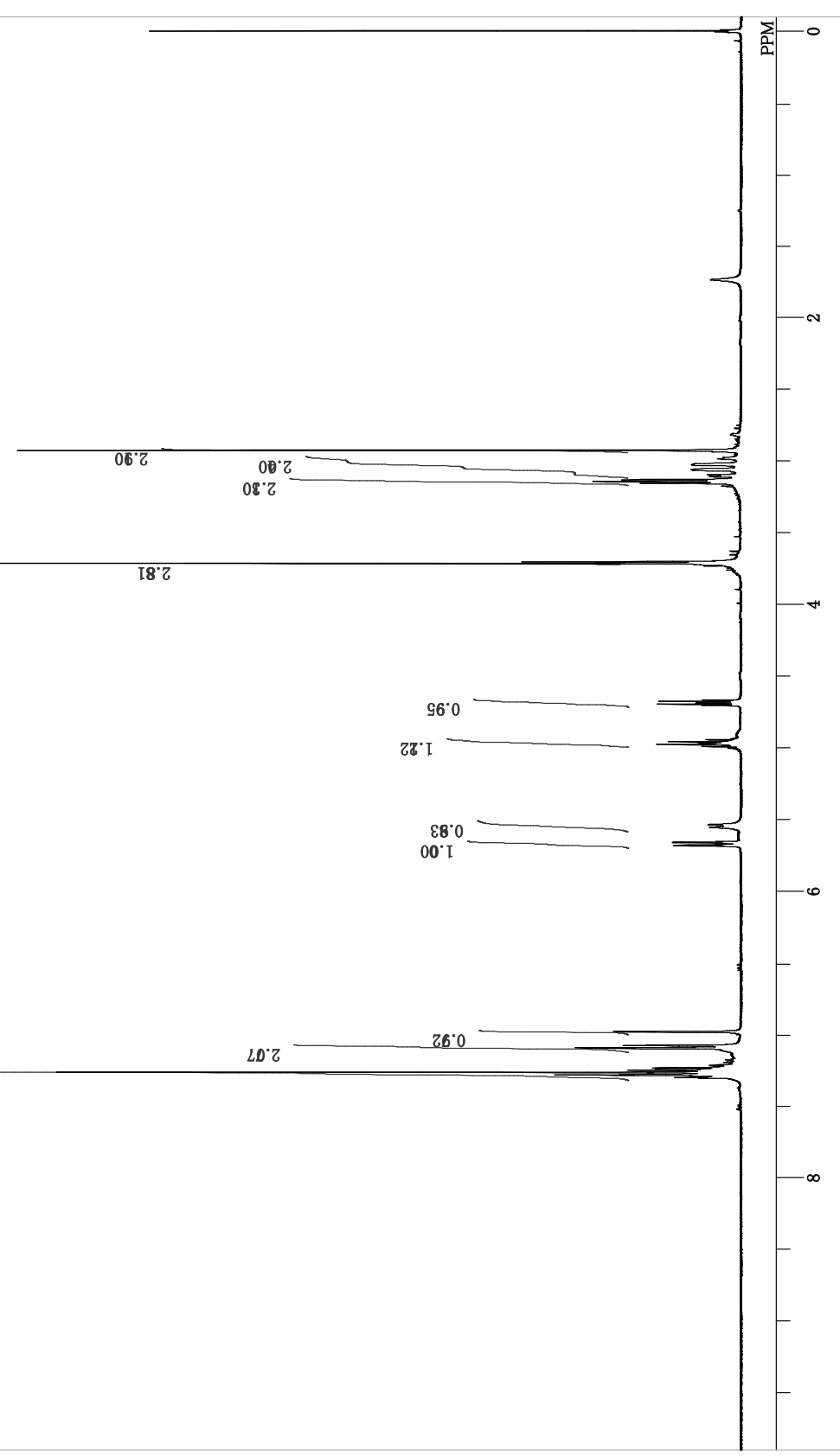



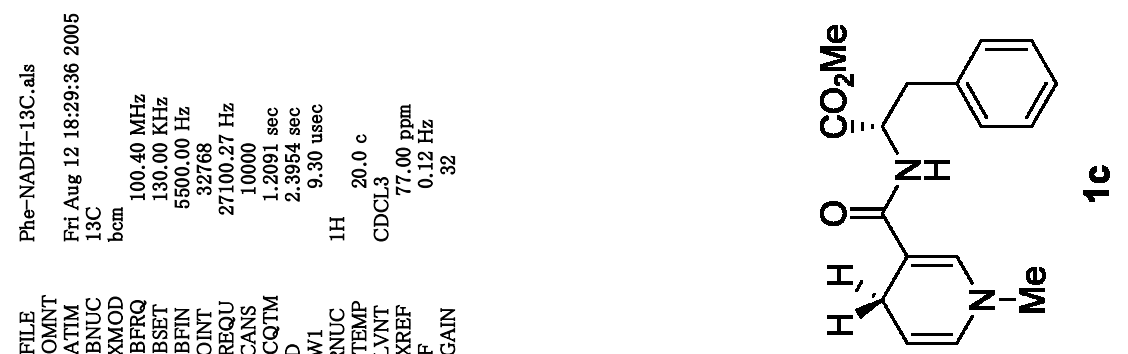

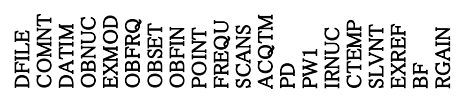

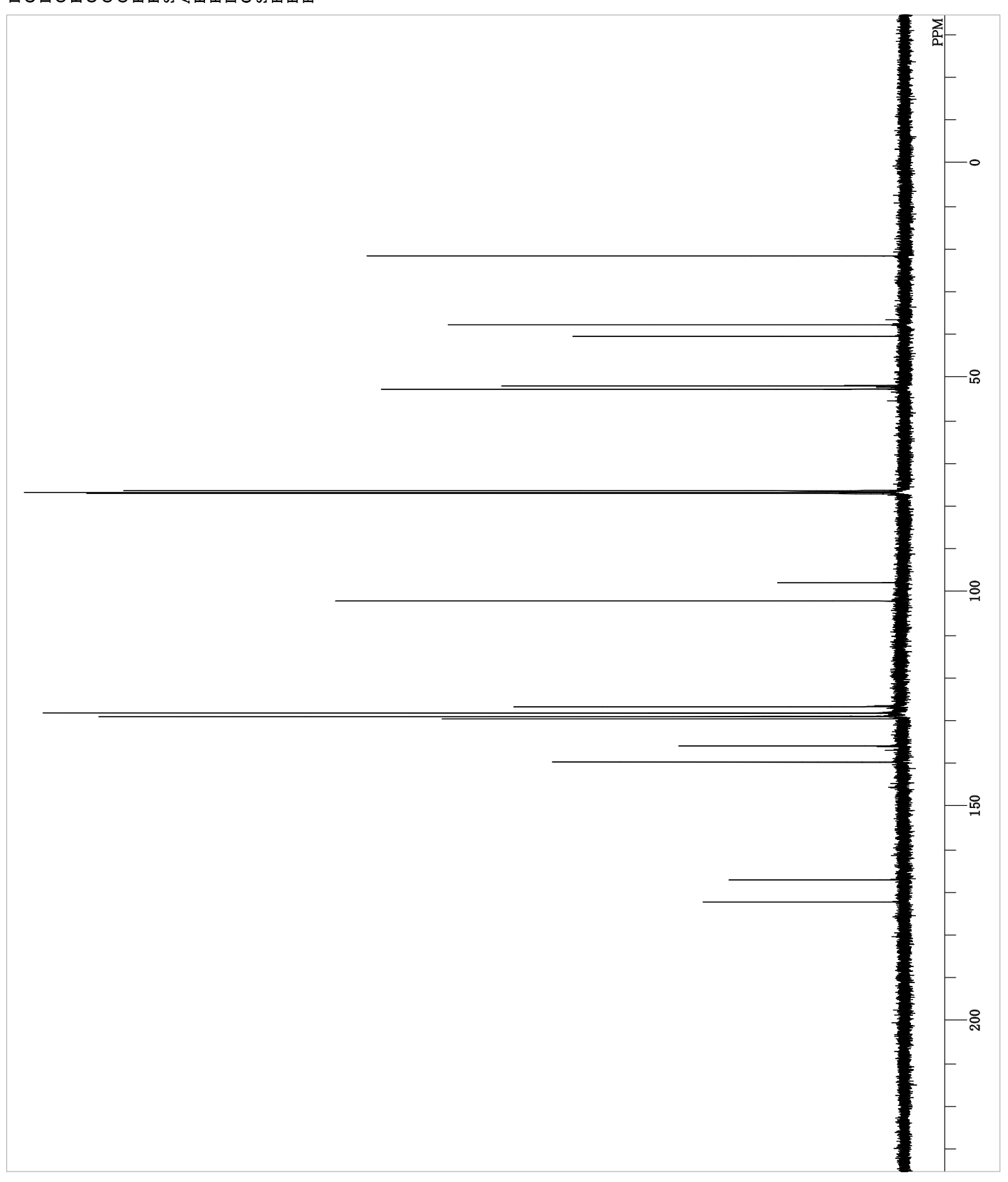




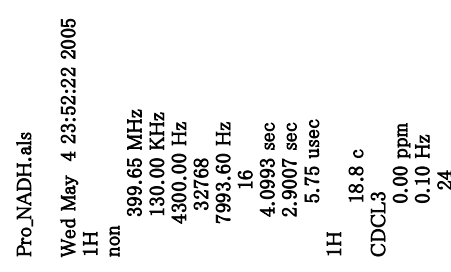

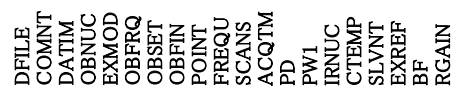
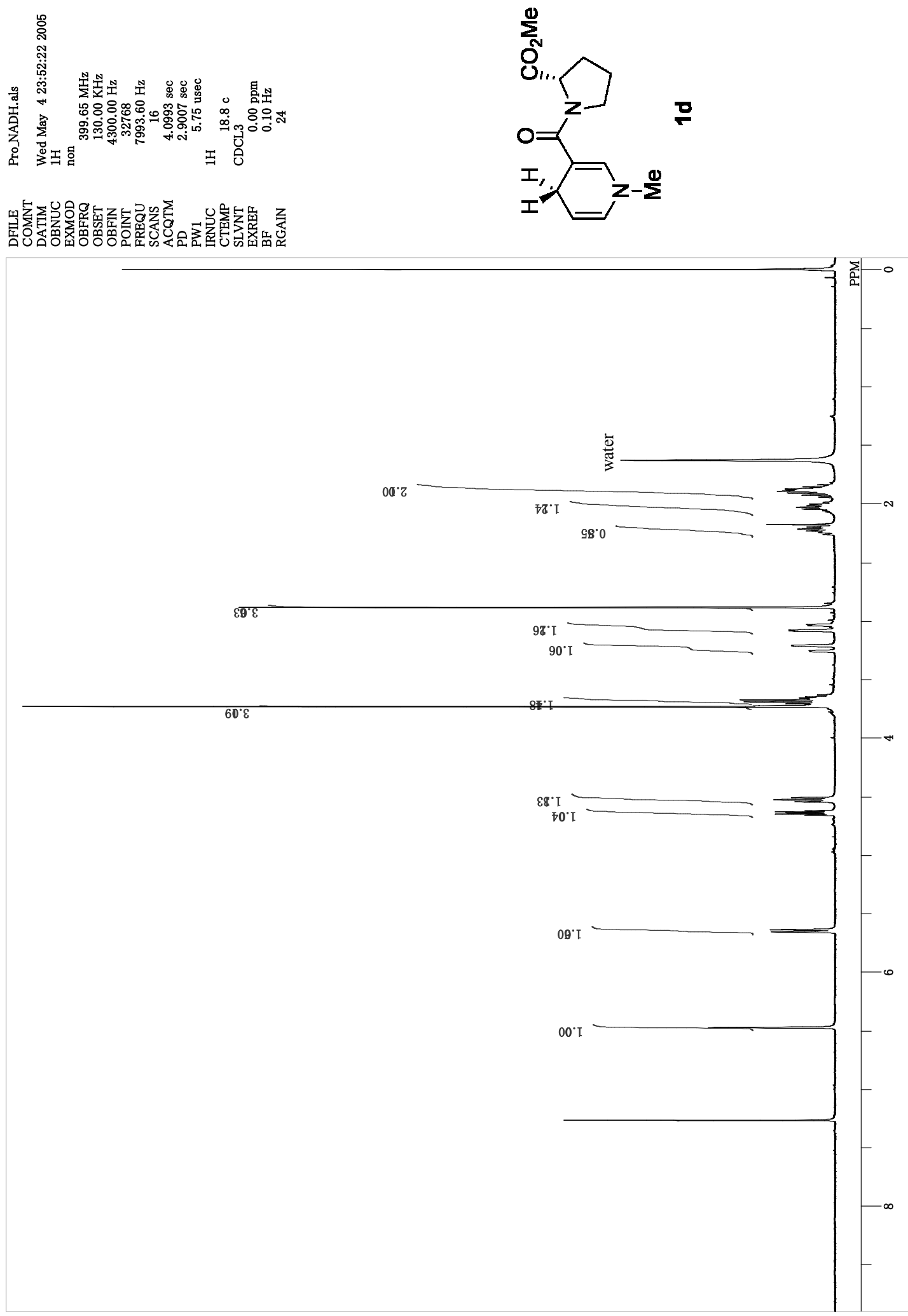


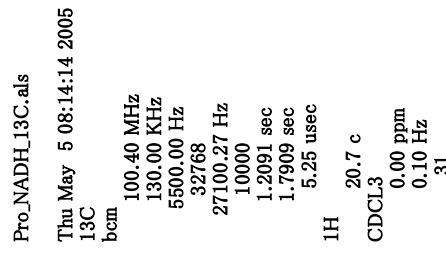

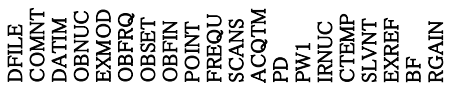
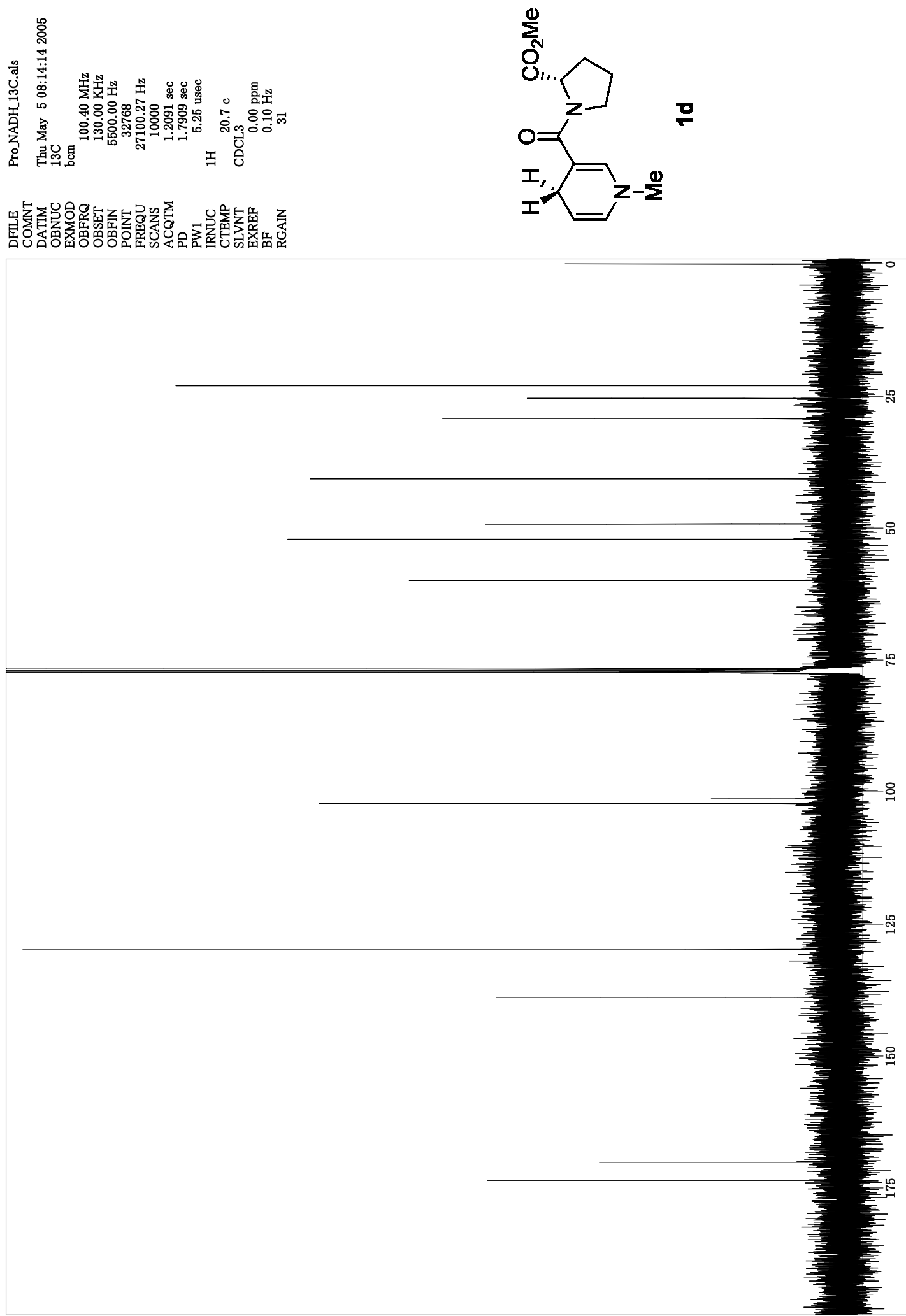


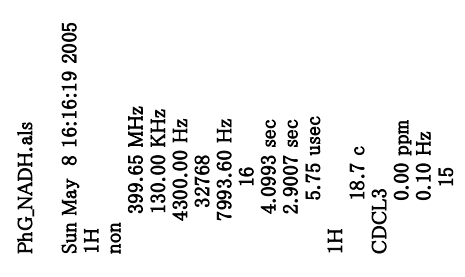

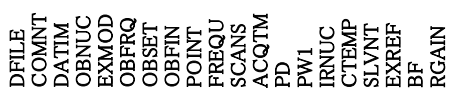
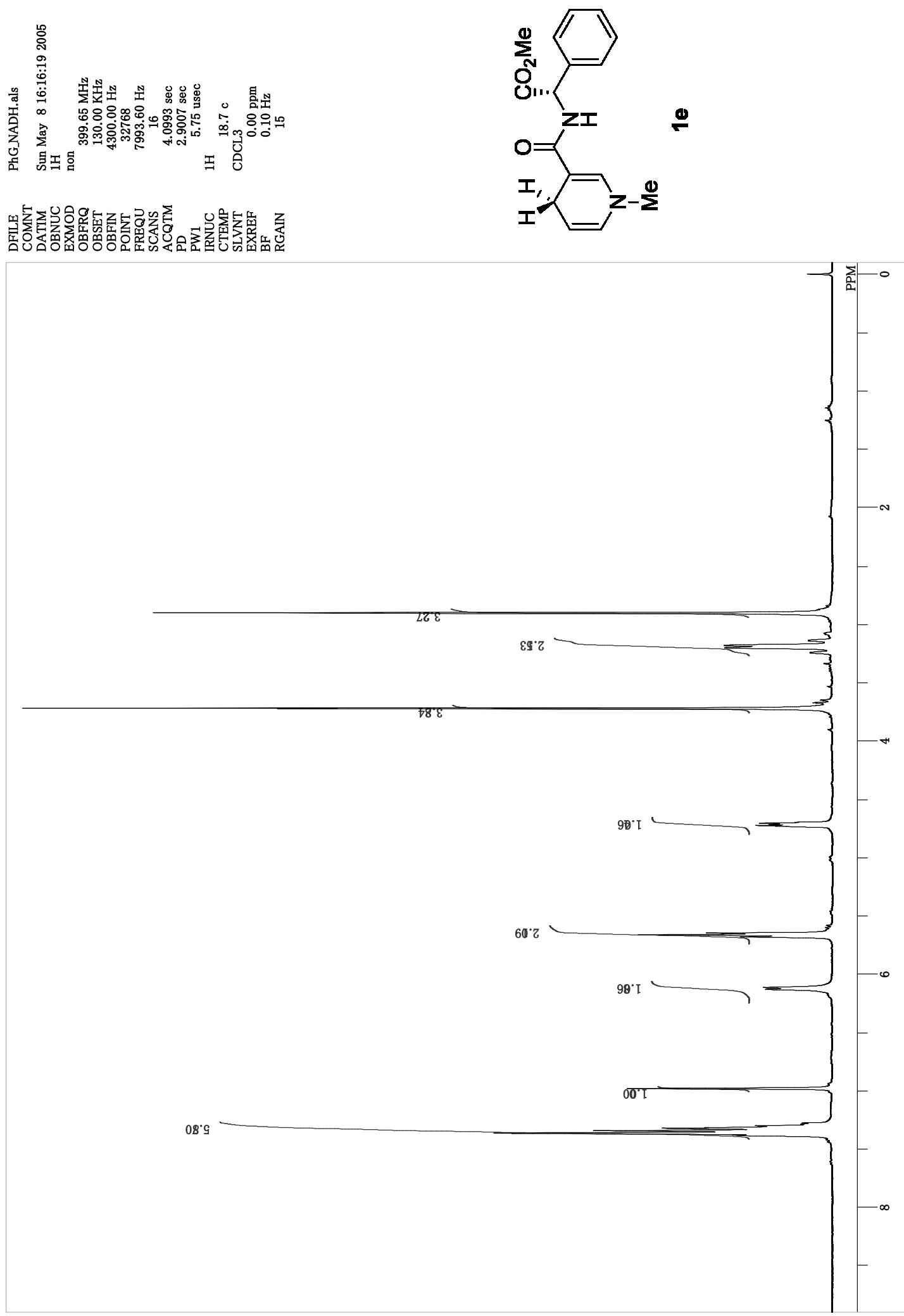


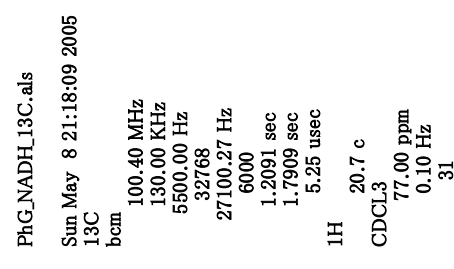

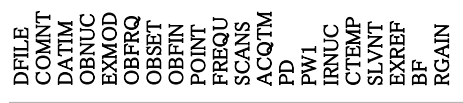
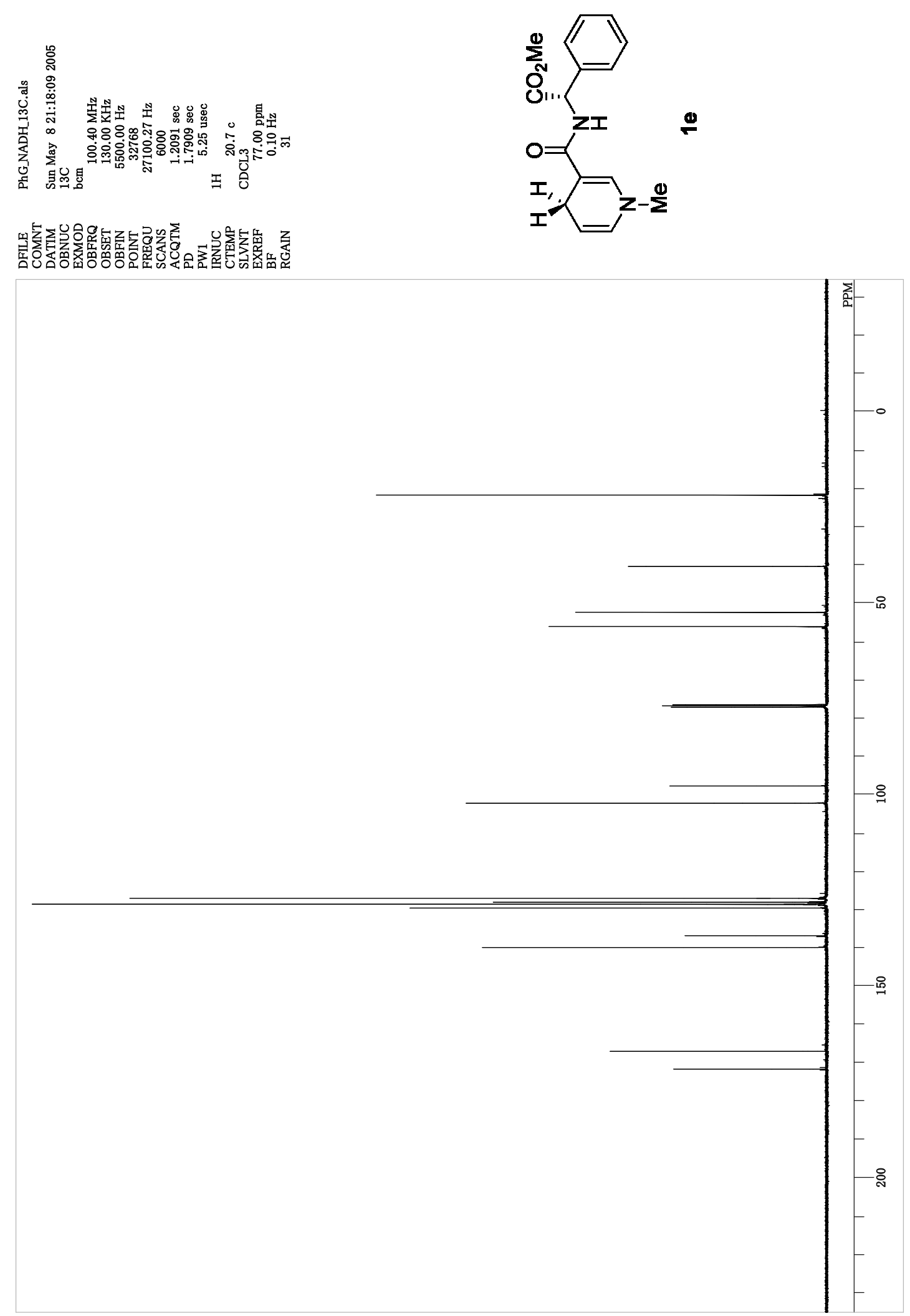